\title{
Thalidomide combined with irradiation alters the activity of two proteases
}

\author{
ECE ŞIMŞEK $^{1}$, ESRA AYDEMIR $^{2}$, AYLIN FIDAN KORCUM $^{3}$ and KAYAHAN FIŞKIN ${ }^{2}$ \\ ${ }^{1}$ Department of Nutrition and Dietetics, Akdeniz University, Antalya School of Health; ${ }^{2}$ Department of Biology, \\ Akdeniz University, Science Faculty; ${ }^{3}$ Department of Radiation Oncology, Akdeniz University, \\ Medicine Faculty, Antalya 07058, Turkey
}

Received January 2, 2014; Accepted October 1, 2014

DOI: $10.3892 / \mathrm{mmr} .2014 .2831$

\begin{abstract}
The aim of the present study was to investigate the effects of thalidomide, a drug known for its anti-angiogenic and antitumor properties, at its cytotoxic dose previously determined as $40 \mu \mathrm{g} / \mathrm{ml}$ (according to four cytotoxic test results). The effect of the drug alone and in combination with radiotherapy using Cobalt $60\left({ }^{60} \mathrm{Co}\right)$ at $45 \mathrm{~Gy}$ on the enzymatic activity of substance-P degrading A disintegrin and metalloproteinase (ADAM)10 and neprilysin (NEP) was investigated in the mouse breast cancer cell lines 4T1 and 4T1 heart metastases post-capsaicin (4THMpc). Thalidomide $(40 \mu \mathrm{g} / \mathrm{ml})$ exerted differing effects on the activities of ADAM10 and NEP enzymes. In 4T1 cells, $40 \mu \mathrm{g} / \mathrm{ml}$ thalidomide alone did not alter ADAM10 enzyme activity. ${ }^{60} \mathrm{Co}$ irradiation at $45 \mathrm{~Gy}$ alone caused a $42 \%$ inhibition in ADAM10 activity, however, the inhibition increased to $89 \%$ when combined therapy was used. By contrast, in the 4THMpc cell line, $40 \mu \mathrm{g} / \mathrm{ml}$ thalidomide alone induced a $66.6 \%$ increase in ADAM10 enzyme activity. Radiotherapy alone and thalidomide with ${ }^{60} \mathrm{Co}$ combined therapy caused a 33.3 and $40 \%$ inhibition of ADAM10 activity, respectively. In 4T1 cells, thalidomide alone caused a $40.9 \%$ increase in NEP activity. Radiation therapy alone or in combination with the drug caused a $40.7 \%$ increase in NEP activity. In more aggressive 4THMpc cells, thalidomide alone caused a $26.6 \%$ increase in NEP activity. Radiotherapy alone and combined therapy caused a 33.3 and $37 \%$ increase in enzyme activity, respectively. To the best of our knowledge, the present study is the first to demonstrate that thalidomide alone or in combination with radiotherapy exhibits significant cytotoxic effects on 4T1 and 4THMpc mouse breast cancer cell lines indicating that this drug affects the enzymatic activity of ADAM10 and NEP in vitro.
\end{abstract}

Correspondence to: Dr Ece Şimşek, Department of Nutrition and Dietetics, Akdeniz University, Antalya School of Health, Antalya 07058, Turkey

E-mail: ecesimsek@akdeniz.edu.tr

Key words: 4T1, 4T1 heart metastases, thalidomide, neprilysin, A disintegrin and metalloproteinase-10

\section{Introduction}

Thalidomide, a derivative of glutamic acid that exists as an equal mixture of its enantiomers was first introduced in Europe for the treatment of morning sickness in pregnant females. Due to its teratogenicity, it was subsequently withdrawn in the late 1960s (1). However, in 1994, D'Amato et al (2) suggested that thalidomide inhibited limb development by inhibiting angiogenesis via the inhibition of basic fibroblast growth factor (bFGF) and/or vascular endothelial growth factor (VEGF). Following this study, Kenyon et al (3) demonstrated that thalidomide inhibited bFGF- and VEGF-induced corneal neovascularization in mice. Bauer et al (4) revealed that thalidomide inhibits microvessel formation in a rat aortic ring angiogenesis assay and slows human aortic endothelial cell proliferation. Furthermore, thalidomide has been demonstrated to inhibit tumor necrosis factor- $\alpha$ (TNF- $\alpha$ ) synthesis by inducing TNF- $\alpha$ mRNA degradation, inhibiting the activation of nuclear factor $\kappa \mathrm{B}(\mathrm{NF}-\kappa \mathrm{B})$ through a mechanism involving the inhibition of I $\kappa \mathrm{B}$ kinase activity and reducing the level of free radicals that cause oxidative DNA damage (5). The anti-angiogenic activity of thalidomide suggested that it may be effective in the treatment of solid tumors and other angiogenesis-related diseases, as angiogenesis in solid tumors is vital for advanced tumor growth and metastasis (6). Currently, thalidomide is one of the most well known teratogens in medical history and clinically recognized as an efficient therapeutic agent for different types of cancer, however, the anti-angiogenic mechanism of thalidomide remains to be elucidated.

Angiogenesis refers to the formation of new blood vessels from the endothelium of existing vasculature; this process is fundamental for tumor growth, progression and metastasis. In previous years, the inhibition of angiogenesis has been demonstrated as a promising strategy for the treatment of cancer and has been successfully transferred from preclinical to clinical applications (7). In the 1970s, Folkman hypothesized that targeting the blood supply by inhibiting blood vessel formation may lead to arrest of tumor growth or even tumor shrinkage. The physiological basis of this hypothesis is that tumors cannot exceed $1-2 \mathrm{~mm}^{3}$ in an avascular state. Following this, intensive and successful studies investigating the molecular mechanisms of tumor angiogenesis were initiated (8). 
The angiogenic switch is considered to be controlled by a balance between pro- and anti-angiogenic molecules in the solid tumor microenvironment. The switch occurs as a result of a net balance of positive and negative regulators. When pro-angiogenic factors overcome the effect of anti-angiogenic molecules, the tumor acquires an angiogenic phenotype that leads to the formation of new blood vessels (9).

Cancer cells can stimulate angiogenesis by producing several angiogenic factors, including VEGF, angiopoetins, bFGF, epidermal growth factor, interleukin 8 and transforming growth factor $\beta$ (7). The presence of angiogenic factors alone is not sufficient to initiate new vascular growth. Pro-angiogenic factors are counterbalanced by a number of natural anti-angiogenic molecules, including thrombospondin-1, angiostatin and endostatin (10). Anti-angiogenic substances can be divided into agents that directly target endothelial cell recruitment, endothelial cell proliferation or tube formation, whereas indirect inhibitors target tumor cell production of pro-angiogenic growth factors or interfere with their receptors or intracellular signaling pathways (11). Multiple studies have reported that thalidomide has anti-angiogenic properties, however, the underlying mechanisms remain to be elucidated (12).

The angiogenesis-related peptide, substance $\mathrm{P}(\mathrm{SP})$ is a member of the tachykinin family, encoded by the preprotachykinin A (PPT-A) 1 gene (13). SP is a major neuropeptide involved in neurogenic inflammation, however, it is also the most significant neuropeptide in cancer. The PPT-A gene is expressed in a number of other cell types, including monocytes, human fibroblasts, keratinocytes, lymphocytes, platelets and tumor cells. SP also induces angiogenesis and local inflammatory responses, which may increase cancer progression and metastases (14). SP appears to exert a bidirectional effect on inflammation, tumor growth and carcinogenesis; these effects may be due to the counter-balancing effects of SP fragments and the intact peptide, such that intact peptide is tumorigenic and induces inflammation, whereas the fragments produced by peptidases are antitumorigenic and anti-angiogenic (15).

The peptidases, neprilysin (NEP) and A disintegrin and metalloproteinase (ADAM)10 degrade SP $(16,17)$. NEP is a membrane-bound, 90-110 kDa, zinc-dependent metallopeptidase that cleaves amide bonds on the amino side of hydrophobic amino acids and is normally expressed in a wide range of tissues and cells. Decreased NEP activity has been linked to increased growth of certain cancer cells, however, whether its activity is associated with the growth of cells has yet to be elucidated (18).

Erin et al (17) revealed that ADAM10 is a multifunctional, membrane-bound, cell surface glycoprotein, which has numerous functions in cell growth, differentiation and motility. The authors demonstrated that ADAM10 hydrolyzes SP; specifically, ADAM10 produces the same growth-inhibitory products as SP (i.e. SP 1-7), similarly to NEP.

It is well established that solid tumors frequently contain a substantial number of cells that are hypoxic. Harrison et al (19) reported that the hypoxia exists in tumors. Numerous studies have demonstrated that hypoxic cells are common in the majority of solid tumors, including malignant brain tumors, melanomas, soft tissue sarcomas, prostate cancer, cervical cancer and invasive breast cancer (20-25). In a clinical setting, particularly for the treatment of breast cancer, radiotherapy can be successfully used in combination with other treatments, including chemotherapy, however, the precise mechanism is not clear (26). For this reason, it is important to determine the effects of thalidomide plus radiotherapy as a combined therapy.

Our initial study demonstrated the cytotoxic effects of thalidomide alone and in combination with radiotherapy in mouse breast cancer cell lines 4T1 and 4T1 heart metastases post-capsaicin (4THMpc) using four cytotoxic tests (data unpublished). Based on these findings, the present study was designed to investigate the association between SP degrading two enzymes and treatment with thalidomide alone or thalidomide in combination with radiotherapy.

Based on the results, it is hypothesized that thalidomide may activate NEP and ADAM10 enzymatic activity in vitro and this process may be responsible for its anti-angiogenic properties.

\section{Materials and methods}

Cell culture. The 4T1 breast cancer cell line, which was originally derived from a breast carcinoma that arose spontaneously in a Balb-c mouse and the 4THMpc cell line derived from cardiac metastases of 4T1 cells were used in the present study. The two cell lines were provided by Dr Nuray Erin at Akdeniz University, Medicine Faculty (Antalya, Turkey).

The 4T1 and 4THMpc cell lines were grown in Dulbecco's modified Eagle's medium (DMEM)/F12 (Invitrogen Life Technologies, Carlsbad, CA, USA) supplemented with $5 \%$ fetal bovine serum (FBS), $2 \mathrm{mM}$ L-glutamine, $1 \mathrm{mM}$ sodium pyruvate and $0.02 \mathrm{mM}$ non-essential amino acids at $37^{\circ} \mathrm{C}$ with $5 \%$ $\mathrm{CO}_{2}$ in a humidified atmosphere. Confluent cells (90\%) were used in all experiments. All cell lines used in the present study were assessed and demonstrated to be free of mycoplasma contamination.

Determination of the cytotoxic dose. The cytotoxic effect of thalidomide alone or in combination with radiotherapy on 4T1 and 4THMpc mouse breast cancer cell lines was determined via four cytotoxic assessments. The viability of the control and treated cells was determined using an MTT colorimetric assay (Promega Corporation, Madison, WI, USA), the trypan blue dye exclusion method (Sigma, St. Louis, MO, USA) and the LIVE/DEAD ${ }^{\circledR}$ cell viability assay (Invitrogen Life Technologies) after 24, 48 and $72 \mathrm{~h}$ of incubation. An EnzCheck ${ }^{\circledR}$ caspase-3 enzyme activity assay (Invitrogen Life Technologies) was performed to determine whether thalidomide causes apoptosis.

Determination of ADAM10 enzyme activity. Confluent cells were trypsinized in a solution of trypsin-versen and resuspended in DMEM/F12 (Biochrom AR, Berlin, Germany) medium with 5\% FBS (Biochrom). Cells were subsequently seeded into $100 \times 20 \mathrm{~mm}$ petri dishes at a density of $3 \times 10^{5}$ cell $/ \mathrm{ml}$. After $24 \mathrm{~h}, 40 \mu \mathrm{g} / \mathrm{ml}$ thalidomide was added and after a further $4 \mathrm{~h}$, radiotherapy labeled petri dishes were irradiated with $45 \mathrm{~Gy}{ }^{60} \mathrm{Co}$. Cells were incubated for an additional $24 \mathrm{~h}$.

Following the incubation period, media was removed and cells were harvested using $1 \mathrm{ml}$ sterile phosphate-buffered 
saline (PBS) and centrifuged at $1,720 \mathrm{x}$ g for $2 \mathrm{~min}$. An assay buffer $(200 \mu \mathrm{l})$ containing $0.1 \%(\mathrm{v} / \mathrm{v})$ Triton-X 100 was added to each cell pellet, incubated at $4^{\circ} \mathrm{C}$ for $10 \mathrm{~min}$ and subsequently centrifuged for $10 \mathrm{~min}$ at $3,100 \mathrm{x} \mathrm{g}$. The supernatant was collected and the protein content of samples was determined using the protein assay kit (Bio-Rad, Hercules, CA, USA) with bovine serum albumin as standard based on the Bradford method. Samples were stored at $-80^{\circ} \mathrm{C}$ prior to use.

The ADAM10 enzyme activity was determined with a fluorimetric assay using a SensoLyte ${ }^{\mathrm{TM}} 520$ TACE $(\alpha$-Secretase) Activity Assay kit (Ana Spec Inc., San Jose, CA, USA) according to the manufacturer's instructions. A sterile black 96 well plate was used for measuring the fluorescence intensity. Each sample contained $100 \mu \mathrm{g} / \mathrm{ml}$ protein and TNF- $\alpha$ converting enzyme substrate solution to a total volume of $100 \mu \mathrm{l}$, which was added to wells according to test procedure. The fluorescence intensities were measured at a wavelength of 495/510 $\mathrm{nm}$ (excitation/emission) with a fluorescence/luminescence spectrometer (Model LS55; PerkinElmer Ltd., Beaconsfield, UK).

Western blot analysis of ADAM10. The alterations in ADAM10 protein expression were analyzed by standard western blotting techniques. Briefly, $25 \mu \mathrm{g}$ of homogenate protein was separated on a $10 \%$ polyacrylamide gel by SDS-PAGE and then transferred onto polyvinylidene difluoride membranes (Hybond-P, Amersham Pharmacia Biotech, Piscataway, NJ, USA) with a semi-dry transfer apparatus. The membranes were blocked with Tris-buffered saline (TBS)-5\% milk and incubated at room temperature with a 1:1,000 dilution of a rabbit polyclonal anti-ADAM10 antibody (cat no. AB19026; Millipore, Billerica, MA, USA). The primary antibody was detected with a 1:10,000 dilution of an anti-mouse peroxidase-conjugated secondary antibody (sc-2005; Santa Cruz Biotechnology, Inc., Santa Cruz, CA, USA) and the blots were visualized using a chemiluminescent substrate (ECL Plus; GE Healthcare, Amersham, UK) and exposure to film (Sigma Aldrich).

Determination of NEP enzyme activity. NEP enzyme activity was measured using a fluorometric assay for the generation of free dansyl-D-Ala-Gly (DAG) from $N$-dansyl-Ala-Gly-D-nitro-Phe-Gly (DAGNPG), the substrate for NEP. Confluent cells were trypsinized using Trypsin-versen and resuspended in DMEM/F12 (Biochrom) medium with 5\% FBS (Biochrom). Cells were subsequently seeded into $100 \times 20 \mathrm{~mm}$ petri dishes at a density of $3 \times 10^{5} \mathrm{cell} / \mathrm{ml}$. After $24 \mathrm{~h}, 40 \mu \mathrm{g} / \mathrm{ml}$ thalidomide was added and following a further $4 \mathrm{~h}$, radiotherapy-labeled petri dishes were irradiated with $45 \mathrm{~Gy}{ }^{60} \mathrm{Cobalt}$. Cells were incubated for an additional $24 \mathrm{~h}$. Following the incubation period, media was removed and cells were mechanically harvested using $500 \mu \mathrm{l}$ sterile PBS and a cell scraper (BD Falcon ${ }^{\mathrm{TM}}$; BD Biosciences, Franklin Lakes, NJ, USA). Cells were homogenized in $250 \mu \mathrm{l}$ of $50 \mathrm{mM}$ Tris-HCl buffer $\mathrm{pH} 7.4$. The homogenate was centrifuged at $1,000 \mathrm{x} \mathrm{g}$ for $10 \mathrm{~min}$ at $4^{\circ} \mathrm{C}$ to remove crude debris and the supernatant was reserved as a sample for the assay. Substrate solutions consisting of $1 \mathrm{mM}$ DAGNPG and $10 \mathrm{mM}$ enalapril in $50 \mathrm{mM}$ Tris- $\mathrm{HCl}$ in the absence and presence of $10 \mathrm{mM}$ phosphoramidon were prepared. The substrate solutions were preincubated at $37^{\circ} \mathrm{C}$ for $10 \mathrm{~min}$. The samples $(50 \mu \mathrm{l})$ were subsequently incubated at $37^{\circ} \mathrm{C}$ for $10 \mathrm{~min}$ with $100 \mathrm{ml}$ of each substrate solution. The reaction was stopped by incubating for $10 \mathrm{~min}$ at $90^{\circ} \mathrm{C}$. The samples were then diluted 1:10 with $50 \mathrm{mM}$ Tris- $\mathrm{HCl}$ and spun for $5 \mathrm{~min}$ in a microfuge at $13,190 \mathrm{x} \mathrm{g}$. The fluorescence of the supernatant was measured using a LS 55 fluorescence spectrophotometer (Perkin-Elmer Inc.) at an emission wavelength of $562 \mathrm{~nm}$ and an excitation wavelength of $342 \mathrm{~nm}$. Samples and DAG standards were assayed in triplicate. The protein concentration of the samples was determined using a Bio-Rad protein assay kit (Bio-Rad) and measured against bovine serum albumin standards.

Western blot analysis for NEP. In order to elucidate whether changes in NEP activity in 4T1 and 4THMpc cells were due to changes in NEP protein content, cell homogenates were analyzed using standard western blotting techniques (27). The membranes were subsequently blocked with TBS-5\% milk and then incubated at room temperature with a 1:2,000 dilution of a rabbit polyclonal antibody known to react with mouse NEP (cat no. AB5458; Millipore). The primary antibody was detected with 1:20,000 dilution of an anti-mouse peroxidase-conjugated secondary antibody (sc-2005; Santa Cruz Biotechnology, Inc.) and the blots were visualized using a chemiluminescent substrate (ECL Plus; GE Healthcare) and exposure to film (Sigma Aldrich).

Statistical analysis. All data are presented as the mean \pm standard error of the mean. Data analysis was performed using INSTAT version 3.0 software (Graph Pad Software, San Diego, CA, USA). Analysis of variance (ANOVA) with Dunnett's multiple comparisons post-hoc test and Student's t-test (for comparisons between two groups) were used for intergroup comparisons. Statistical analyses for the SP quantity were performed using either ANOVA followed by the Tukey-Kramer multiple comparison test or Student's paired t-test on the percentage of alteration values. The graphs were drawn using Sigma Plot version 10.0 (SPSS, Inc., Chicago, IL, USA) and CorelDRAW version X4 (Corel, Co., Minneapolis, MN, USA). $\mathrm{P}<0.05$ was considered to indicate a statistically significant difference.

\section{Results}

Determining the cytotoxic effects of thalidomide alone or in combination with radiotherapy. In our previous study, 10 concentrations of thalidomide and different fractions of irradiation $\left(0,0001-40 \mu \mathrm{g} / \mathrm{ml}\right.$ thalidomide and 5-45 $\left.\mathrm{Gy}{ }^{60} \mathrm{Co}\right)$ were used to determine the cytotoxic effects of the drug alone or in combination with radiotherapy (data not shown). According to four cytotoxicity test results, $40 \mu \mathrm{g} / \mathrm{ml}$ thalidomide alone or in combination with $45 \mathrm{~Gy}{ }^{60} \mathrm{Co}$ irradiation exhibits the most significant cytotoxic effect on 4T1 and 4THMpc cell lines. The optimum combination for enzyme activity and SP experiments were selected to be $40 \mu \mathrm{g} / \mathrm{ml}$ thalidomide and $45 \mathrm{~Gy}{ }^{60} \mathrm{Co}$ irradiation.

Determination of ADAM10 enzyme activity. The effects of $40 \mu \mathrm{g} / \mathrm{ml}$ thalidomide alone or in combination with $45 \mathrm{~Gy}{ }^{60} \mathrm{Co}$ irradiation on ADAM10 enzyme activity in the 4T1 cell 


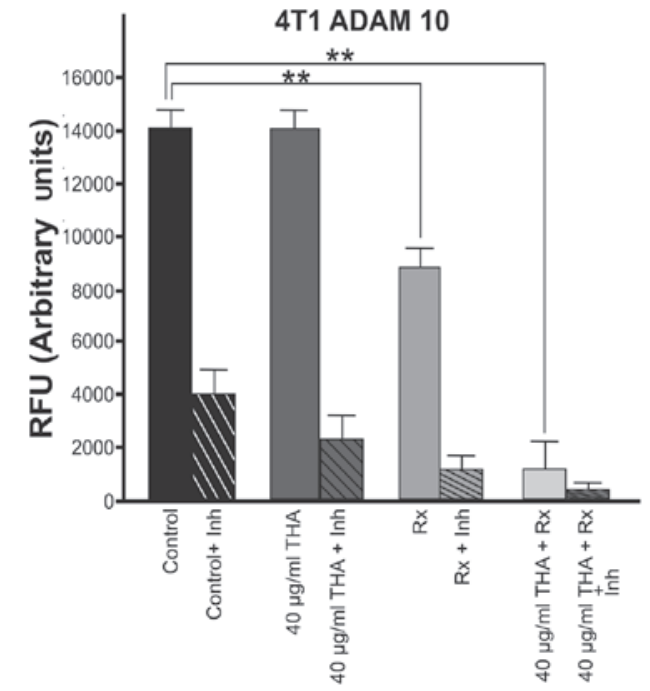

Figure 1. Effects of thalidomide and irradiation on ADAM10 enzyme activity in $4 \mathrm{~T} 1$ cells. ADAM10, A disintegrin and metalloproteinase 10; 4T1HMpc, $4 \mathrm{~T} 1$ heart metastases post-capsaicin.

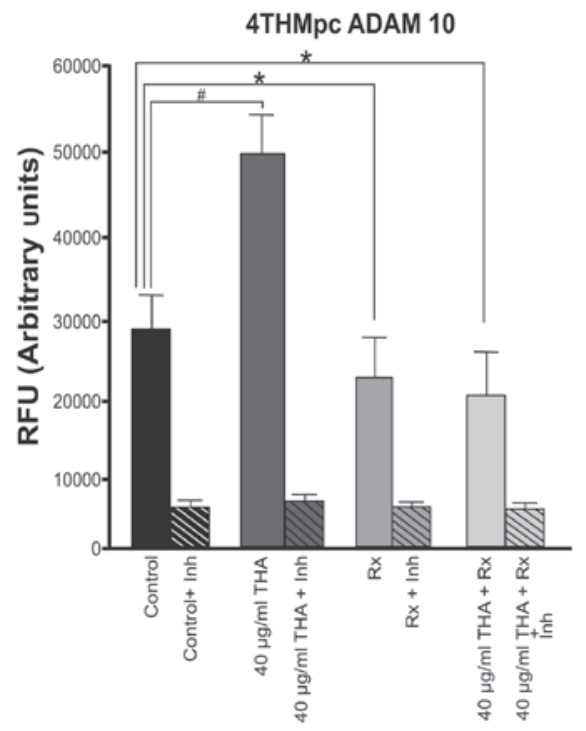

Figure 2. Effects of thalidomide and irradiation on NEP enzyme activity in 4T1HMpc cells. NEP, neprilysin; 4T1HMpc, 4T1 heart metastases post-capsaicin.

line is shown in Fig. 1. Thalidomide $(40 \mu \mathrm{g} / \mathrm{ml})$ alone did not alter the basal level of ADAM10 activity in 4T1 cells compared with the control group. By contrast, ${ }^{60} \mathrm{Cobalt}$ irradiation (45 Gy) alone caused a significant decrease in ADAM10 enzyme activity $(\mathrm{P}<0.01)$. Combined therapy also significantly decreased the ADAM10 enzyme activity in this cell line ( $\mathrm{P}<0.01$; Fig. 1).

ADAM10 enzyme activity was higher at a basal level in the 4THMpc cell line compared with 4T1 cells. By contrast to the 4T1 cells, thalidomide increased the activity of the ADAM10 enzyme in 4THMpc cells. In the 4THMpc cell line, thalidomide increased the activity of the ADAM10 enzyme in the control group. However, ${ }^{60} \mathrm{Co}$ irradiation (45 Gy) alone or in combination with $40 \mu \mathrm{g} / \mathrm{ml}$ thalidomide decreased the level of ADAM10 enzyme activity to the basal level (Fig. 2).

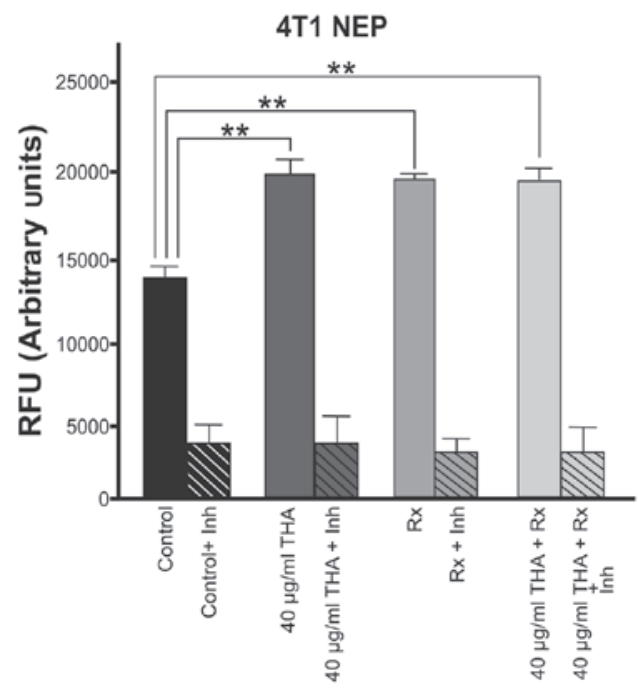

Figure 3. Effects of thalidomide on NEP enzyme activity in 4T1 cells. NEP, neprilysin; RFU, relative fluorescence units; THA, thalidomide; Rx, radiotherapy; Inh, inhibitor of enzyme.

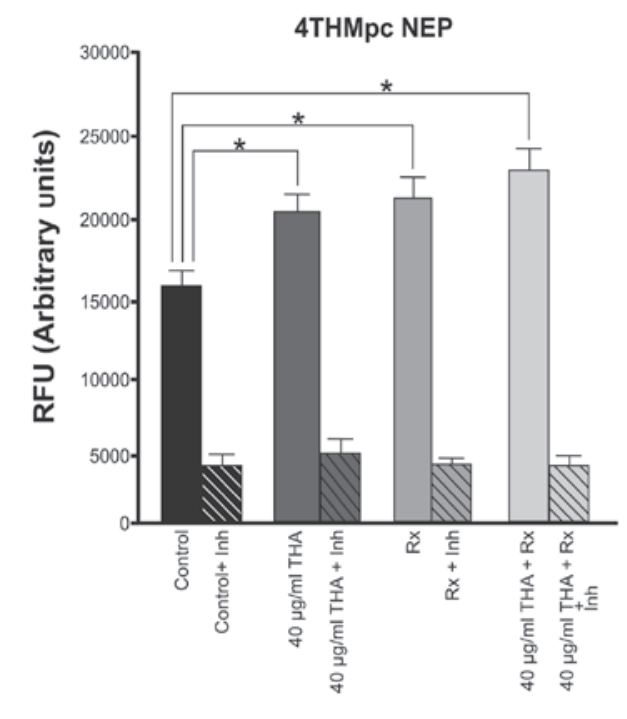

Figure 4. Effects of thalidomide on NEP enzyme activity in 4THMpc cells. NEP, neprilysin; RFU, relative fluorescence units; THA, thalidomide; 4THMpc, 4TI heart metastases post-capsaicin; Rx, radiotherapy; Inh, inhibitor of enzyme.

Determination of NEP enzyme activity. The basal level of NEP enzyme activity was higher in 4T1 cells compared with 4THMpc cells. As shown in Fig. 3, thalidomide and ${ }^{60} \mathrm{Co}$ irradiation alone and the combined therapy caused an approximately equal increase in NEP enzyme activity in 4T1 cells.

In the 4THMpc cell line, thalidomide and ${ }^{60} \mathrm{Cobalt}$ irradiation alone had a similar effect and caused an equal increase in the activity of NEP, however, the enzyme activity was increased further when the combined therapy was used (Fig. 4).

Western blot analysis for ADAM10 and NEP enzymes. To determine whether the changes in NEP and ADAM10 activities of $4 \mathrm{~T} 1$ and $4 \mathrm{THMpc}$ cells were due to alterations in NEP 


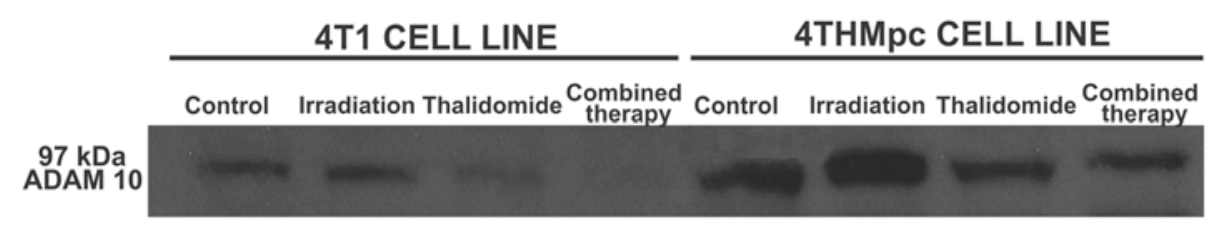

Figure 5. Western blot analyses of A disintegrin and metalloproteinase 10 enzyme. NEP, neprilysin; RFU, relative fluorescence units; THA, thalidomide; Rx, radiotherapy; Inh, inhibitor of enzyme.

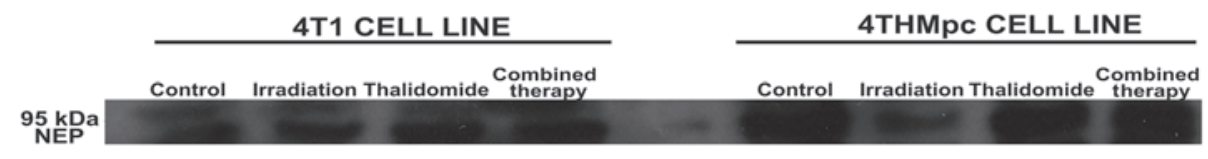

Figure 6. Western blot analyses of the NEP enzyme in 4T1 and 4THMpc cells. NEP, neprilysin; RFU, relative fluorescence units; THA, thalidomide; Rx, radiotherapy; Inh, inhibitor of enzyme.

and ADAM10 protein concentration, cell homogenates were analyzed by standard western blotting techniques. As shown in Figs. 5 and 6, determined enzyme activities were corrected with western blot analysis. The density of the bands correlated with the activities of the two enzymes.

\section{Discussion}

Following the identification of the teratogenic effects of thalidomide in pregnant females, the drug was subsequently demonstrated to destroy blood vessels in the fetus (28). Due to this property, thalidomide was investigated as an anti-angiogenic drug and its potential anti-angiogenic and antitumoral properties have been demonstrated in an animal model (29). Currently, thalidomide is successfully used for the treatment of multiple myeloma, prostate cancer and kidney cancer. Research into the potential use of of thalidomide in other types of cancer continues (30).

Breast cancer is one of the most important global health concerns, with $>1,500,000$ new cases and $>400,000$ mortalities annually, worldwide (27). Thalidomide alone is not effective in the treatment of metastatic breast cancer, therefore, it must be combined with another cytotoxic drug or an alternative treatment (1). For a number of years, combining a cytotoxic agent with radiotherapy has been of interest to oncologists. Radiotherapy is the most widely used treatment, particularly in the treatment of solid tumors. In previous years, significant results for breast cancer patients were derived from the use of postoperative systemic therapies and radiotherapy. Although these two modalities have been extensively used, the exact mechanisms of their effects in combination remain to be elucidated. The aim of the present study was to investigate the effect of thalidomide in combination with radiotherapy on mouse breast cancer cell lines 4T1 and 4THMpc.

Angiogenesis, the formation of new blood vessels by the extension or elaboration of pre-existing blood vessels, is significant in tumor growth, progression and metastasis. The inhibition of tumor angiogenesis is a promising strategy for the treatment of cancer, which has been successfully transferred from preclinical to clinical applications in recent years.
Numerous drugs are utilized clinically as anti-angiogenic agents and act in different pathways in angiogenesis. One of the main targets of anti-angiogenic drugs is to inhibit proteases released from cancer cells. Proteases are a class of enzymes, which degrade proteins, generating peptides and amino acids. Cancer cells release proteases and degrade the extracellular matrix proteins to facilitate migration, metastases and angiogenesis. These proteases are termed matrix metalloproteases (MMPs) (31).

ADAMs are a subfamily of MMPs and exist in multiple organisms from protozoa to humans. ADAMs are critical for multiple signal transduction pathways and degradative membrane proteins alter them to their soluble mature forms. One of the most important ADAMs members is ADAM10 (32). An increase in ADAM10 protein expression is associated with prostate and breast cancer, however, the underlying mechanism remains to be elucidated.

In $4 \mathrm{~T} 1$ cells, thalidomide $(40 \mu \mathrm{g} / \mathrm{ml})$ alone did not alter the activity of ADAM10. ${ }^{60} \mathrm{Co}$ irradiation (45 Gy) alone caused $42 \%$ inhibition in ADAM10 activity and the inhibition increased to $89 \%$ when combined therapy was used. By contrast, in the 4THMpc cell line, thalidomide $(40 \mu \mathrm{g} /$ $\mathrm{ml}$ ) alone induced a $66.6 \%$ increase in ADAM10 enzyme activity. Radiotherapy alone and thalidomide with ${ }^{60} \mathrm{Co}$ combined therapy caused a 33.3 and $40 \%$ inhibition in ADAM10 activity, respectively. These results were supported by western blot analysis.

NEP, also known as neutral endopeptidase, is a metallopeptidase with a zinc-binding motif (33). NEP is an important peptidase in neuropeptide metabolism. NEP has been demonstrated to activate bombesin, calsistonine, neurotensin and vasoactive intestinal peptit and is also important in tumor growth in lung and prostate cancer (34).

In $4 \mathrm{~T} 1$ cells, thalidomide alone caused a $40.9 \%$ increase in NEP activity. Radiation therapy alone or in combination with the drug caused a $40.7 \%$ increase in NEP activity. Therefore, it is suggested that there is no synergistic effect between radiotherapy and thalidomide.

In the more aggressive 4THMpc cells, thalidomide alone led to a $26.6 \%$ increase in NEP activity. Radiotherapy alone and combined therapy caused 33.3 and $37 \%$ increases in enzyme activity, respectively. At the same dosage, thalidomide 
did not affect the activity of ADAM10, however, NEP enzyme activity was increased. This result may assist in elucidating the mechanism of action of the drug. However, in 4THMpc cells, combined therapy did not alter NEP activity. 4THMpc cells are more aggressive than $4 \mathrm{~T} 1$ cells and ADAMs are associated with angiogenesis, thus, the increased activity of ADAM10 in 4THMpc cells compared with 4T1 cells was unexpected.

It is possible that ADAM10 or NEP may degrade SP. SP and its receptor, neurokinin-1, are associated with mitogenesis, angiogenesis, migration and metastases. SP was classified as a neurotransmitter in the 1950s and has an important function in inflammation and cancer $(35,36)$.

Associations between SP and pancreas, breast and colon cancer have been reported. SP exists in neuronal cells and cancer cells (37-39). Based on the hypothesis that all solid tumors require angiogenesis for nutrition, the new blood vessel formation triggered by SP and its receptor is the most important link between angiogenesis and cancer (40). Therefore, eradication of SP or its receptors is a novel strategy for cancer treatment.

According to the present results, thalidomide at its toxic dose was determined to exert a significant effect on the activities of ADAM10 and NEP enzymes in 4T1 and 4THMpc mouse breast cancer cell lines in vitro.

To the best of our knowledge, the present study is the first to demonstrate that thalidomide alters the activities of ADAM10 and NEP enzymes causing significant alterations to the mouse breast cancer cell lines, 4T1 and 4THMpc. It is hypothesized that thalidomide may exert its anti-angiogenic property by altering the activity of the two proteases. However, further studies are required to elucidate this mechanism.

\section{Acknowledgements}

This study was supported by The Scientific and Technological Research Council of Turkey (grant no. 107T204). The authors would like to thank Dr Nina Tuncel and all the technicians in the Radiation Oncology Department for their technical assistance. The authors would also like to thank all employees of Akdeniz University Research Unit under the leadership of Professor Olcay Yeğin for their support during this study. In addition, the authors are appreciative of Professor Bahriye Uğur Yavuzer for her useful comments and suggestions on the results of the western blot analysis and Ms. Duygu Sahintürk Ünal for her excellent technical assistance.

\section{References}

1. Papaiakovou VE, Bamias A and Dimopoulos MA: Thalidomide in cancer medicine. Ann Oncol 15: 1151-1160, 2004.

2. D'Amato RJ, Loughnan MS, Flynn E, et al: Thalidomide is an inhibitor of angiogenesis. Proc Natl Acad Sci USA 91: 4082-4085, 1994.

3. Kenyon BM, Browne F and D'Amato RJ: Effects of thalidomide and related metabolites in a mouse corneal model of neovascularization. Exp Eye Res 64: 971-978, 1997.

4. Bauer KS, Dixon SC and Figg WD: Inhibition of angiogenesis by thalidomide requires metabolic activation, which is species-dependent. Biochem Pharmacol 55: 1827-1834, 1998.

5. Majumdar S, Lamothe B and Aggarwal BB: Thalidomide suppresses NF- $\kappa$ B activation induced by TNF and $\mathrm{H}_{2} \mathrm{O}_{2}$, but not that activated by ceramide, lipopolysaccharides, or phorbol ester. J Immunol 168: 2644-2651, 2002.
6. Macpherson GR, Franks M, Tomoaia-Cotisel A, et al: Current status of thalidomide and its role in the treatment of metastatic prostate cancer. Crit Rev Oncol Hematol 46: 49-57, 2003.

7. Eichhorn ME, Kleespies A, Angele MK, et al: Angiogenesis in cancer: molecular mechanisms, clinical impact. Langenbecks Arch Surg 392: 371-379, 2007.

8. Folkman J, Watson K, Ingber D et al: Induction of angiogenesis during the transition from hyperplasia to neoplasia. Nature 339: 58-61, 1989.

9. Jain RK: Normalizing tumor vasculature with anti-angiogenic therapy: a new paradigm for combination therapy. Nat Med 7: 987-989, 2001.

10. Huang $Z$ and Bao SD: Roles of main pro- and anti-angiogenic factors in tumor angiogenesis. World J Gastroenterol 10: 463-470, 2004.

11. Eichhorn ME, Strieth S and Dellian M: Anti-vascular tumor therapy: recent advances, pitfalls and clinical perspectives. Drug Resist Updat 7: 125-138, 2004.

12. Rajkumar SV: Current status of thalidomide in the treatment of cancer. Oncology (Williston Park) 15: 867-874, 2001.

13. Sumner SC, Gallagher KS and Davis DG: Conformational analysis of the tachykinins in solution: substance $\mathrm{P}$ and physalaemin. J Biomol Struct Dyn 8: 687-707, 1990.

14. Carter MS and Krause JE: Structure, expression and some regulatory mechanisms of the rat preprotachykinin gene encoding substance p, neurokinin a, neuropeptide k, and neuropeptide gamma. J Neurosci 10: 2203-2214, 1990.

15. Erin $\mathrm{N}$ and Ulusoy O: Differentiation of neuronal from non-neuronal Substance P. Regul pept 152: 108-113, 2009.

16. Harrison S and Geppetti P: Substance p. Int J Biochem Cell Biol 33: 555-576, 2001.

17. Erin N, Zhao W, Bylander J, et al: Capsaicin-induced inactivation of sensory neurons promotes a more aggressive gene expression phenotype in breast cancer cells. Breast Cancer Res Treat 99: 351-364, 2006.

18. Erin N, Boyer PJ, Bonneau RH, et al: Capsaicin-mediated denervation of sensory neurons promotes mammary tumor metastasis to lung and heart. Anticancer Res 24: 1003-1009, 2004.

19. Harrison LB, Chadha M, Hill RJ, et al: Impact of tumor hypoxia and anemia on radiation therapy outcomes. Oncologist 7: 492-508, 2002.

20. Rampling R, Cruickshank G, Lewis AD et al. Direct measurement of $\mathrm{pO} 2$ distribution and bioreductive enzymes in human malignant brain tumors. Int J Radiat Oncol Biol Phy29: 427-431, 1994.

21. Lartigau E, Randrianarivelo $\mathrm{H}$, Avril MF, et al: Intratumoral oxygen tension in metastatic melanoma. Melanoma Res 7: 400-406, 1997.

22. Nordsmark M, Hoyer M, Keller J, et al: The relationship between tumor oxygenation and cell proliferation in human soft tissue sarcomas. Int J Radiat Oncol Biol Phys 35: 701-708, 1996.

23. Movsas B, Chapman JD, Greenberg RE, et al: Increasing levels of hypoxia in prostate carcinoma correlate significantly with increasing clinical stage and patient age: an Eppendorf $\mathrm{pO}(2)$ study. Cancer 89: 2018-2024, 2000.

24. Mundt AJ, Connell PP, Campbell T, et al: Race and clinical outcome in patients with carcinoma of the uterine cervix treated with radiation therapy. Gynecol Oncol 71: 151-158, 1998.

25. Vaupel P, Briest $S$ and Höckel M: Hypoxia in breast cancer: pathogenesis, characterization and biological/therapeutic implications. Wein Med Wochenschr 152: 334-342, 2002.

26. Harrison L and Blackwell K: Hypoxia and anemia: factors in decreased sensitivity to radiation therapy and chemotherapy? Oncologist 9: 31-40, 2004.

27. Oz ES, Aydemir E, Korcum AF and Fiskin K: Thalidomide and irradiation combination therapy increases substance P levels in vitro. Exp Ther Med 2: 529-535, 2011.

28. D'Amato RJ, Loughnan MS, Flynn E, et al: Thalidomide is an inhibitor of angiogenesis. Proc Natl Acad Sci USA 91: 4082-4085, 1994.

29. Bartlett JB, Dredge K and Dalgleish AG: The evolution of thalidomide and its IMiD derivatives as anticancer agents. Nat Rev Cancer 4: 314-322, 2004

30. Downs LS Jr, Rogers LM, Yokoyama Y and Ramakrishnan S: Thalidomide and angiostatin inhibit tumor growth in a murine xenograft model of human cervical cancer. Gynecol Oncol 98: 203-210, 2005.

31. Vandeputte-Rutten L and Gros P: Novel proteases: common themes and surprising features. Curr Opin Struc Biol 12: 704-708, 2002. 
32. Chubinskaya S, Mikhail R, Deutsch A and Tindal MH: ADAM-10 protein is present in human articular cartilage primarily in the membrane-bound from and is upregulated in osteoarthrits and in response to IL-1 alpha in bovine nasal cartilage. J Histochem Cytochem 49: 1165-1176, 2001.

33. Turner AJ and Hooper NM: The angiotensin-converting enzyme gene family: genomics and pharmacology. Trends Pharmacol Sci 23: 177-183, 2002.

34. Shipp MA, Richardson NE, Sayre PH, et al: Molecular cloning of the common acute lymphoblastic leukemia antigen (CALLA) identifies a type II integral membrane protein. Proc Natl Acad Sci USA 85: 4819-4823, 1988.

35. Luo W, Sharif TR and Sharif M: Substance P-induced mitogenesis in human astrocytoma cells correlates with activation of the mitogenactivated protein kinase signaling pathway. Cancer Res 56: 4983-4991, 1996.

36. Defea KA,Zalevsky J, Thoma MS, et al: Beta arrestin-dependent endocytosis of proteinase-activated receptor 2 is required for intracellular targeting of activated ERK1/2. J Cell Biol 148: $1267-1281,2000$.
37. Bhatia M, Saluja AK, Hofbauer B, Frossard JL, Lee HS, Castagliuolo I, Wang CC, Gerard N, Pothoulakis C and Steer ML: Role of substance $\mathrm{P}$ and the neurokinin 1 receptor in acute pancreatitis and pancreatitis-associated lung injury. Proc Natl Acad Sci USA 95: 4760-4765, 1998.

38. Garcia-Recio S, Fuster G, Fernandez-Nogueira P, Pastor-Arroyo EM, Park SY, Mayordomo C, Ametller E, Mancino M, Gonzalez-Farre X, Russnes HG, et al: Substance $\mathrm{P}$ autocrine signaling contributes to persistent HER2 activation that drives malignant progression and drug resistance in breast cancer. Cancer Res 73: 6424-6434, 2013.

39. Muñoz M and Coveñas R: Neurokinin-1 receptor: a new promising target in the treatment of cancer. Discov Med 10: 305-313, 2010.

40. Esteban F, Muñoz M, González-Moles MA and Rosso M: A role for substance $\mathrm{P}$ in cancer promotion and progression: a mechanism to counteract intracellular death signals following oncogene activation or DNA damage. Cancer Metastasis Rev 25: $137-145,2006$ 